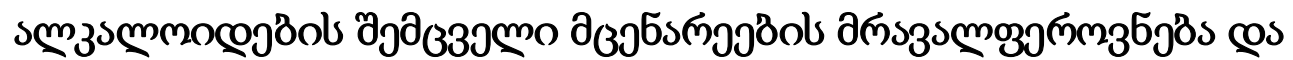

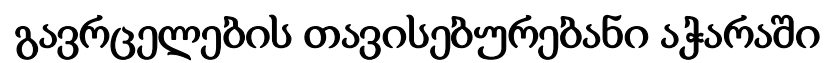

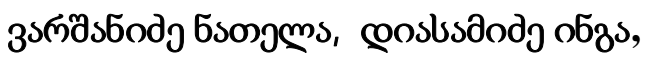

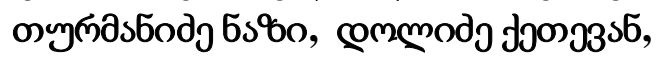

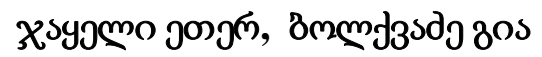

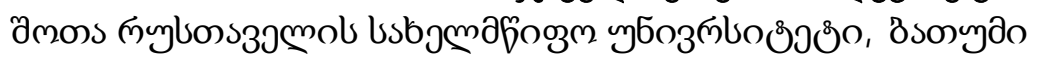

https://doi.org/10.52340/idw .2021.490

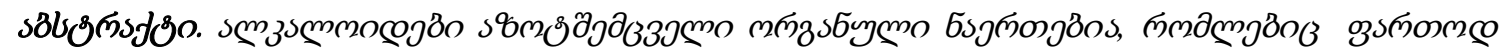

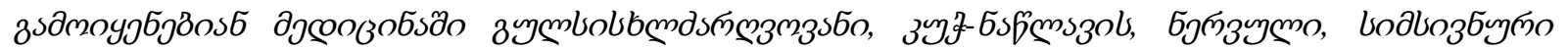

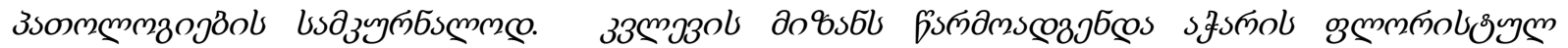

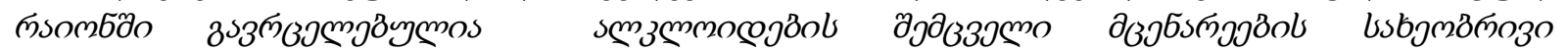

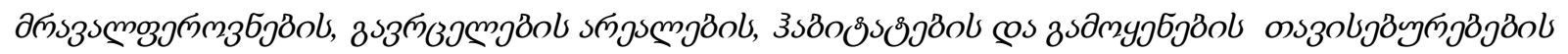

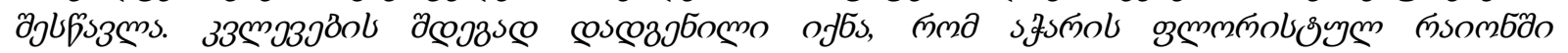

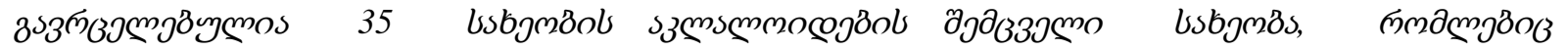

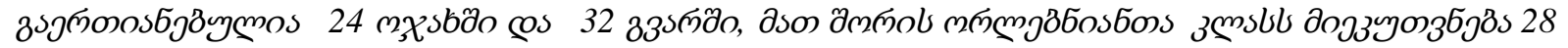

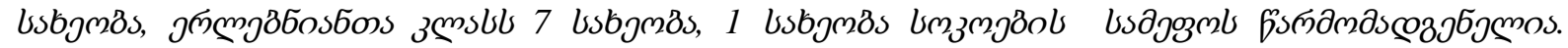

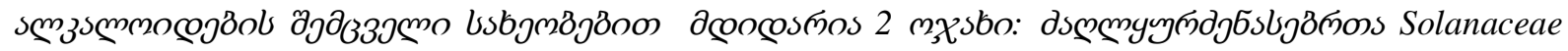

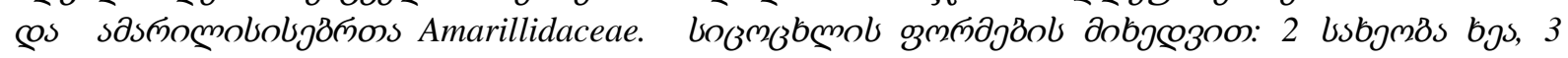

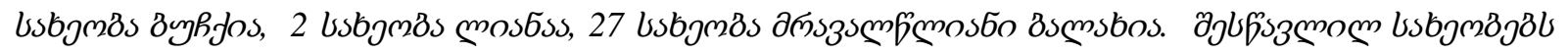

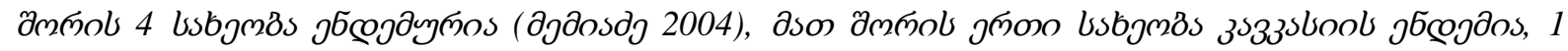

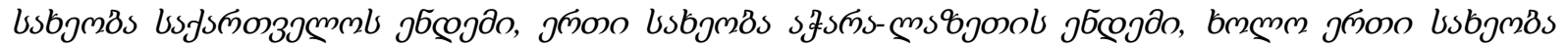

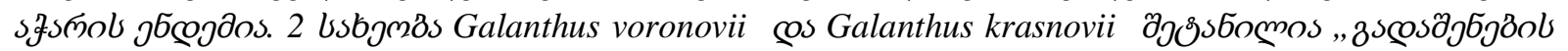

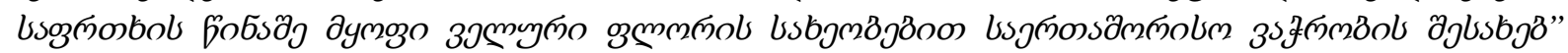

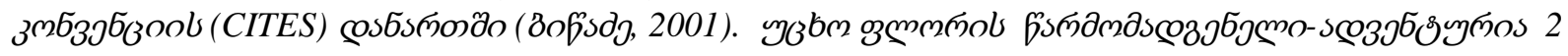

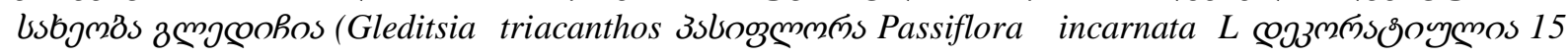

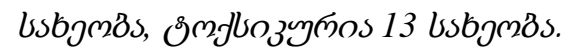

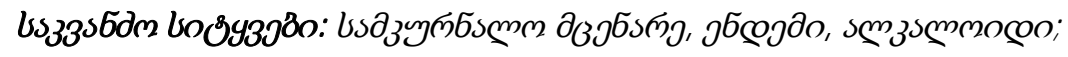

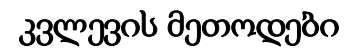

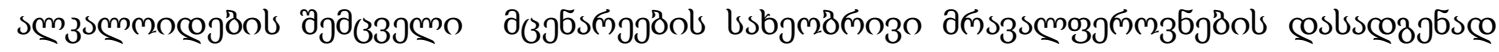

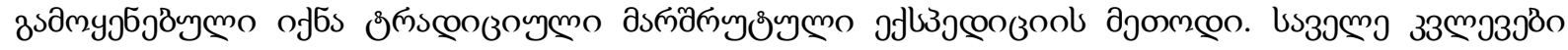

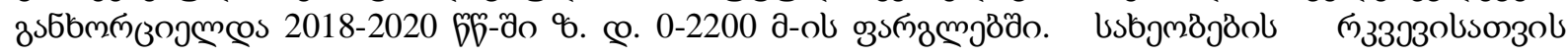

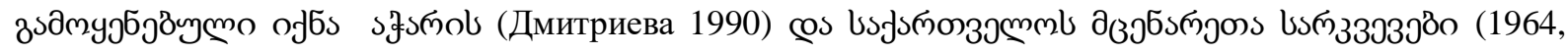

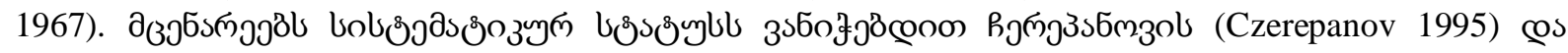

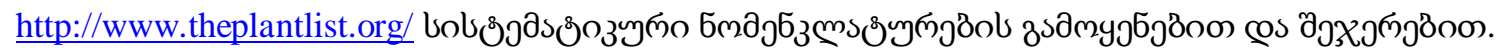

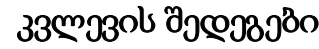

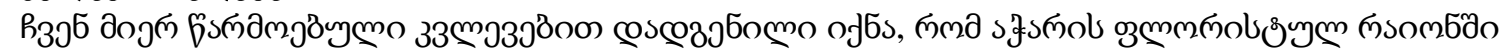

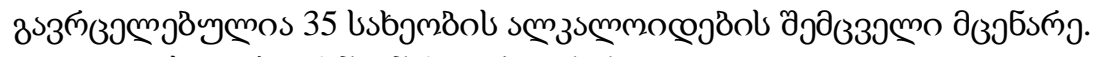

змsbo мпм

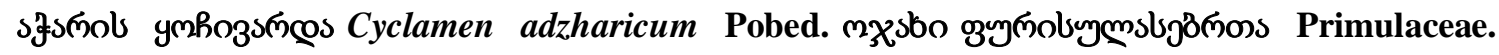

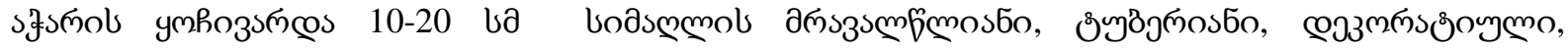

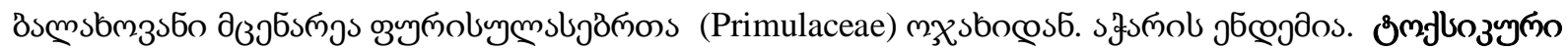




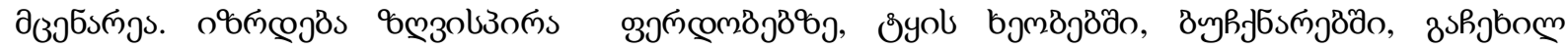

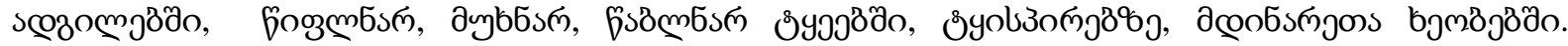

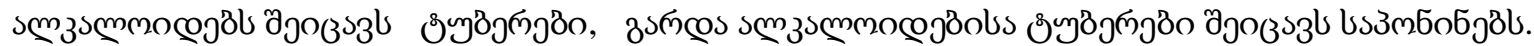

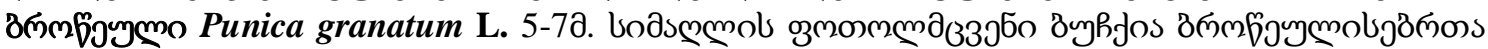

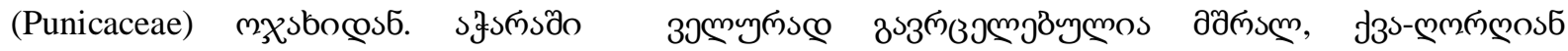

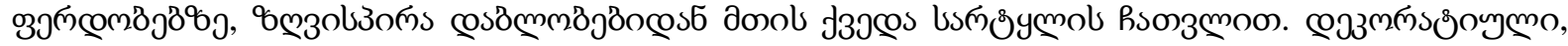

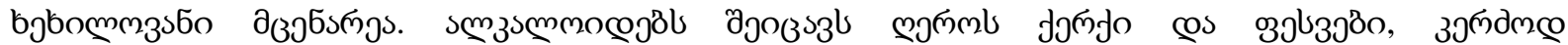

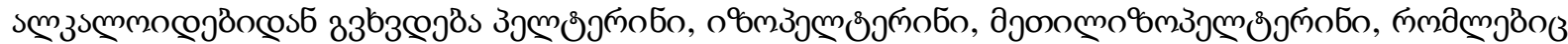

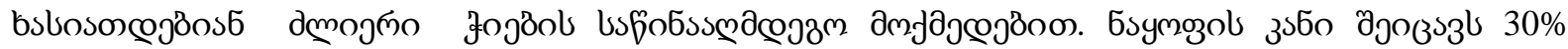

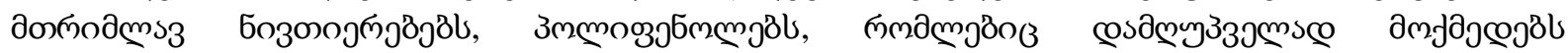

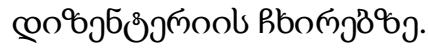

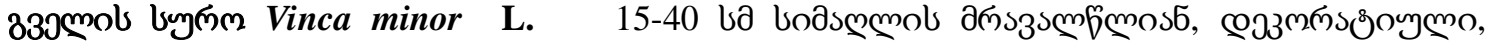

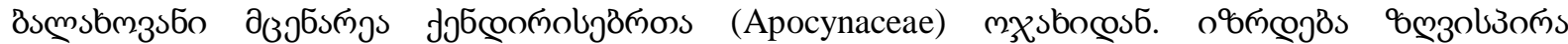

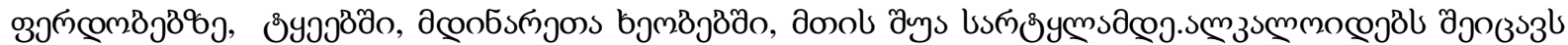

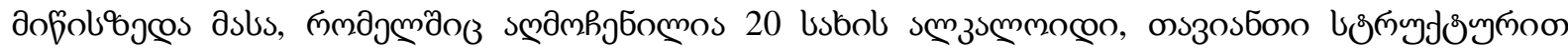

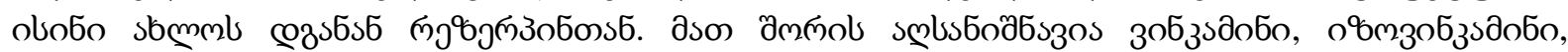

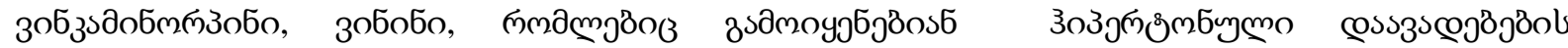
usдзулбблммме.

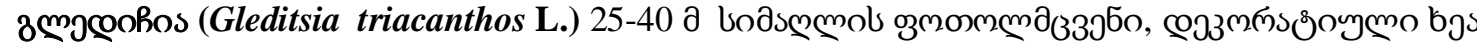

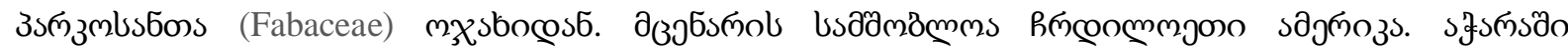

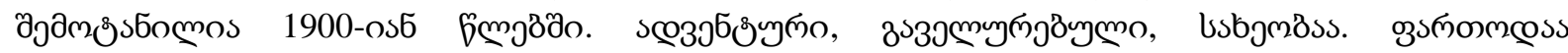

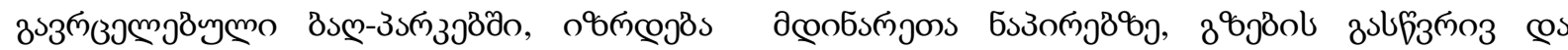

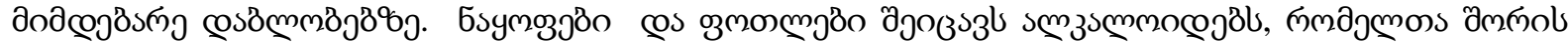

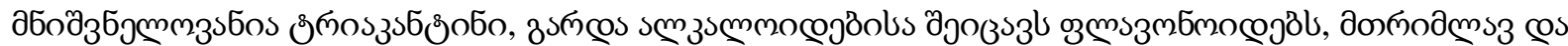

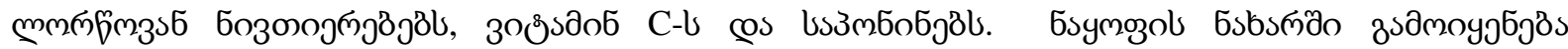

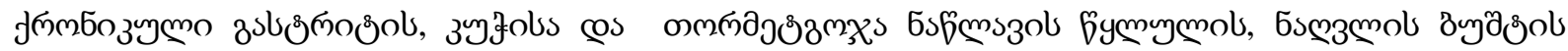

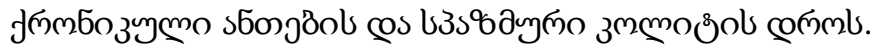

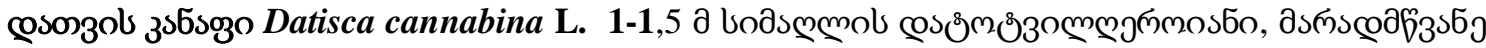

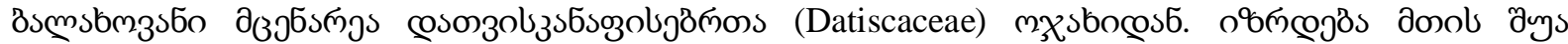

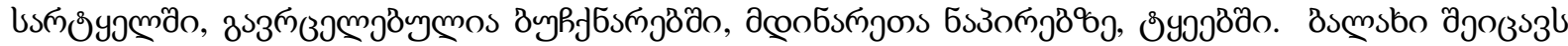

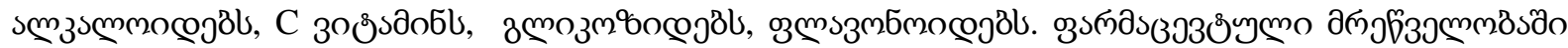

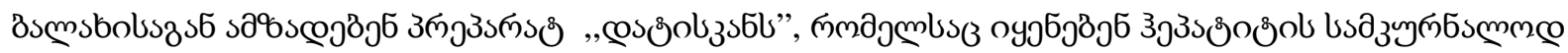

obsfismonbsens, bsmozuges Chamerion angustifolium (L.) Holub. 1,5 a boasलmol

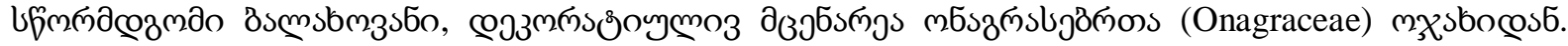

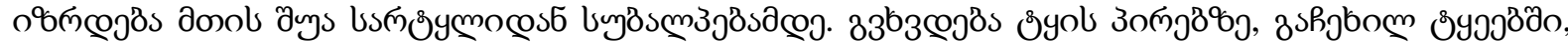

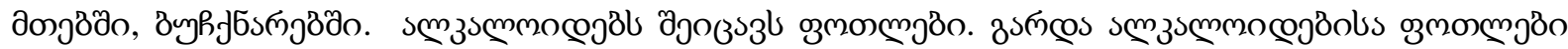

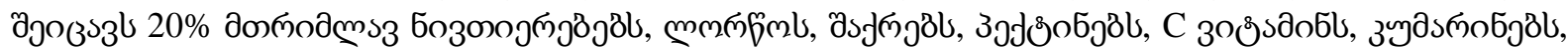

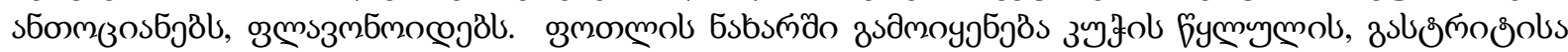
cos zmmos̊ol comml.

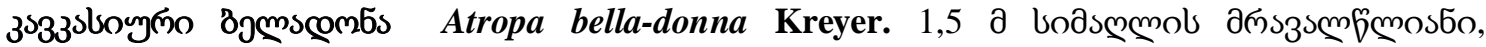

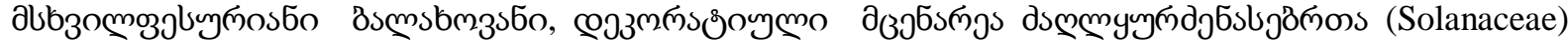

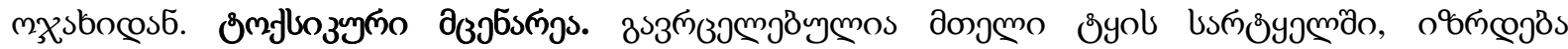

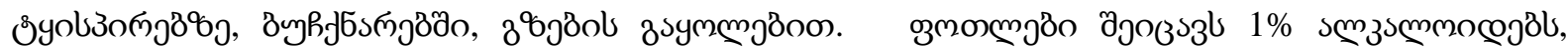

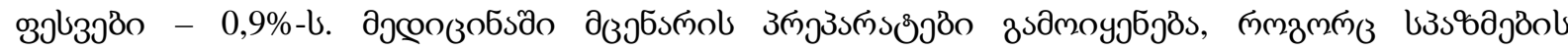

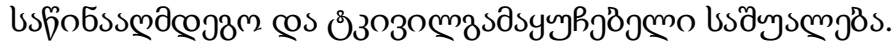

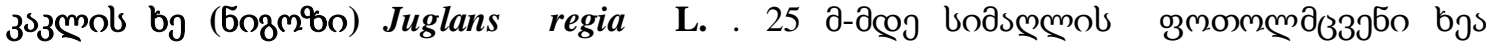

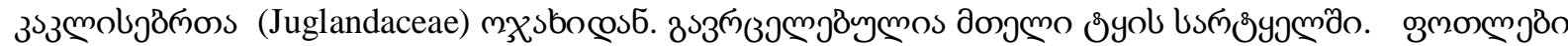

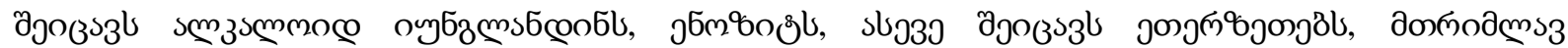

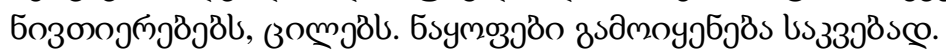

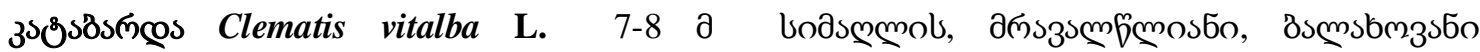




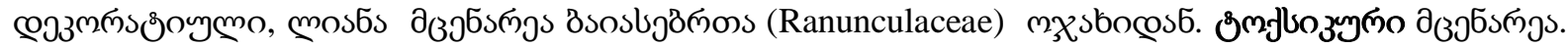

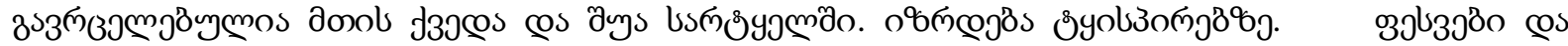

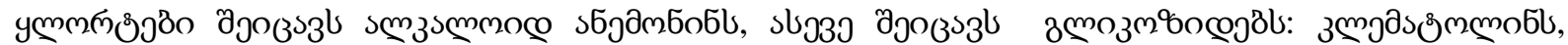

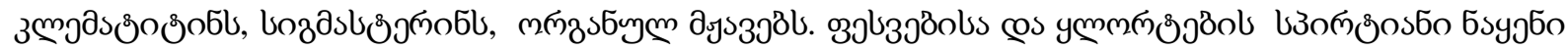

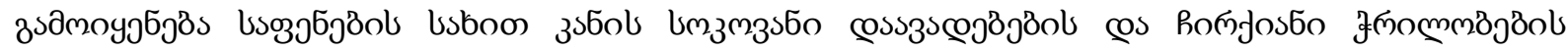

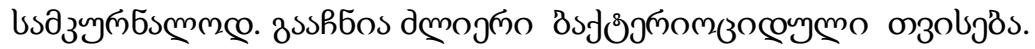

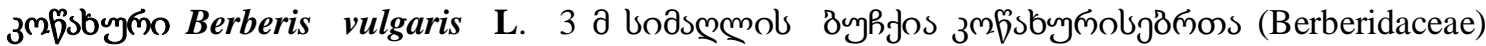

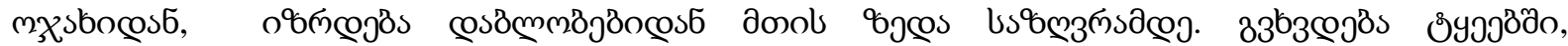

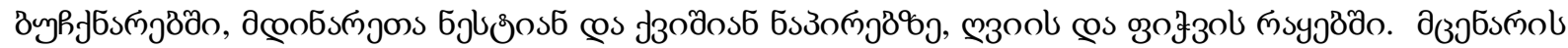

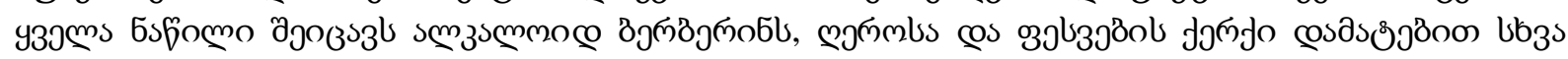

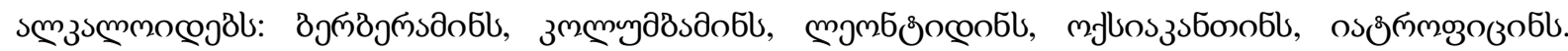

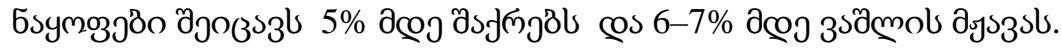

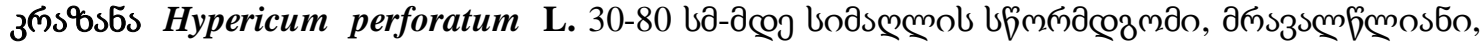

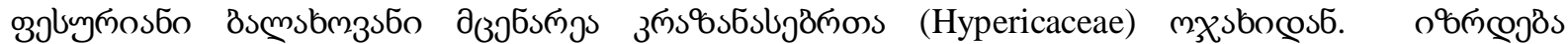

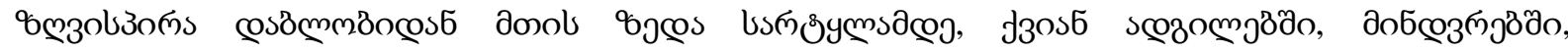

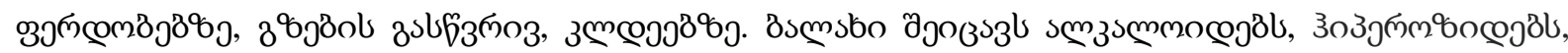

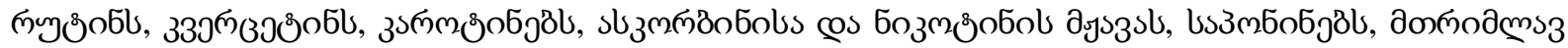

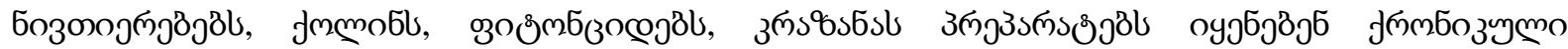

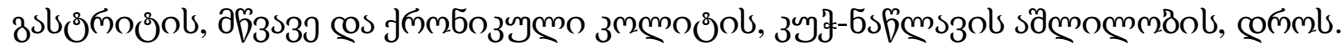

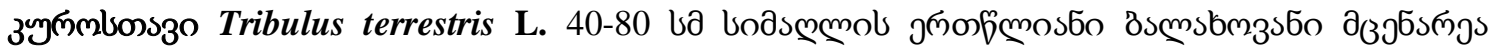

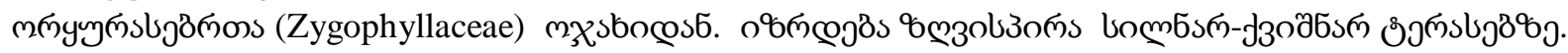

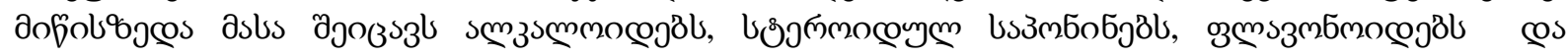

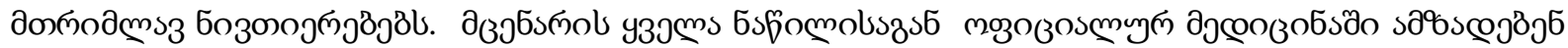

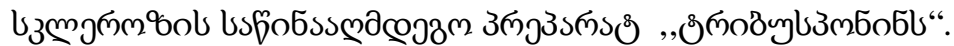

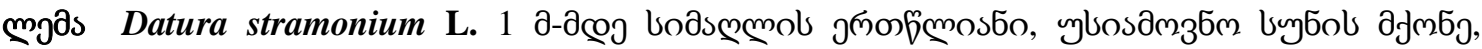

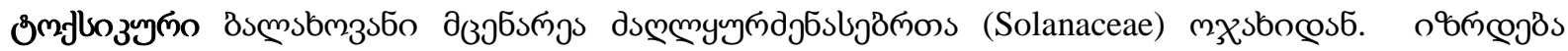

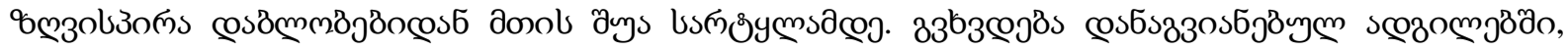

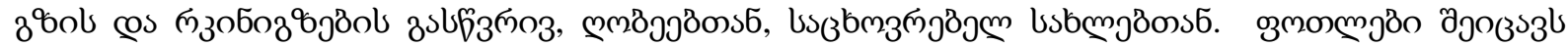

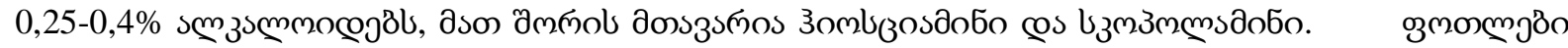

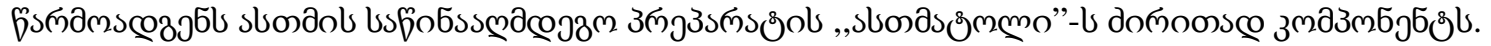

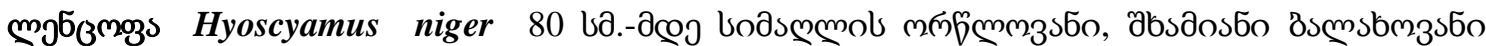

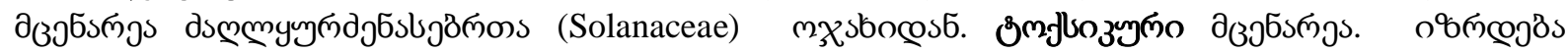

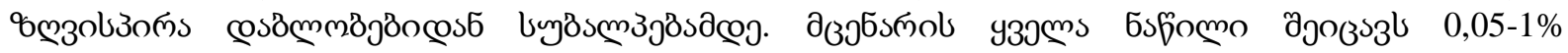

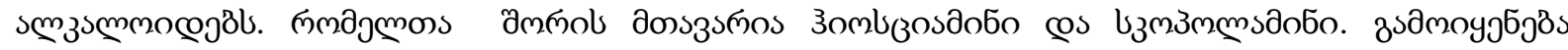

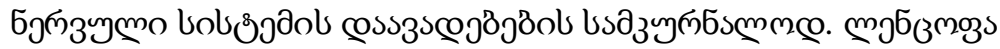

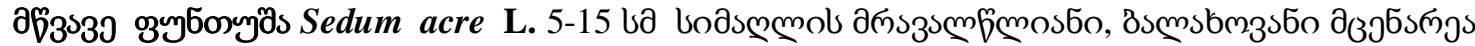

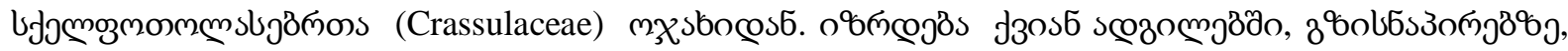

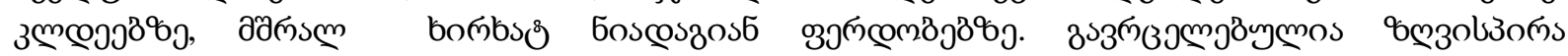

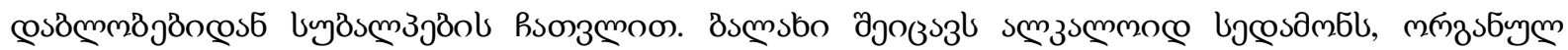

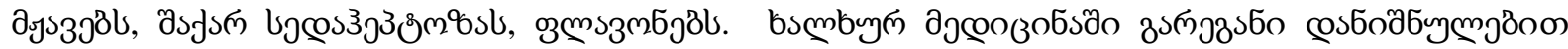

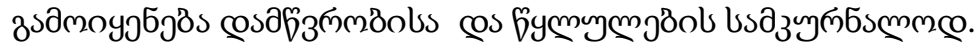

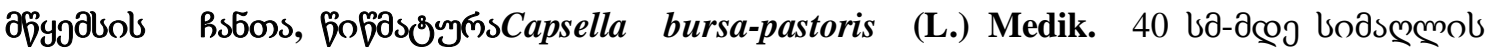

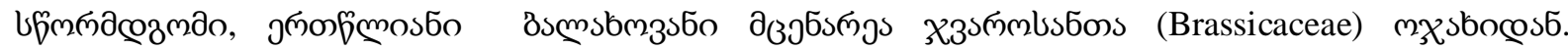

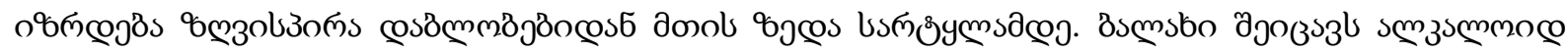

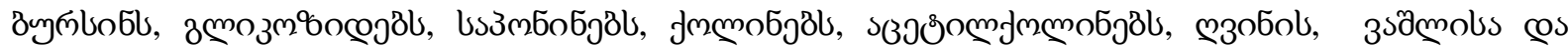

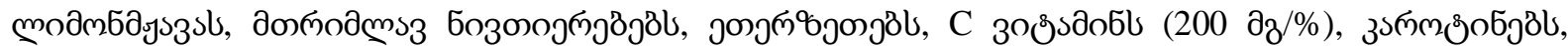

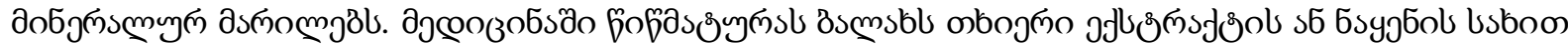

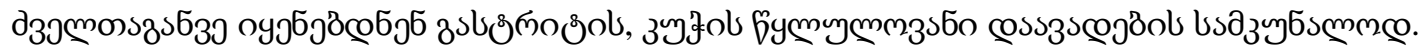

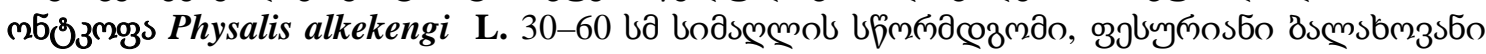

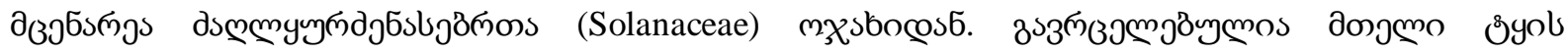




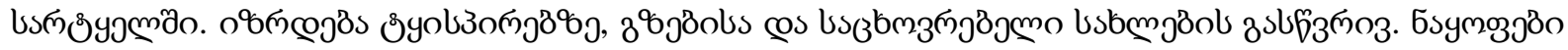

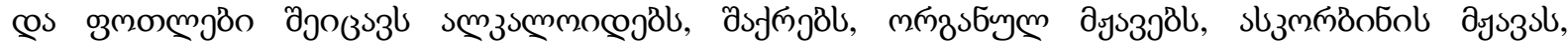

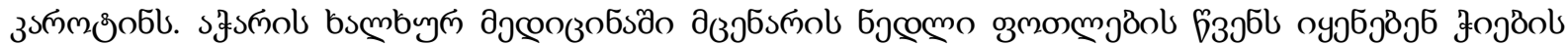
cosacgб bsàysmgissce.

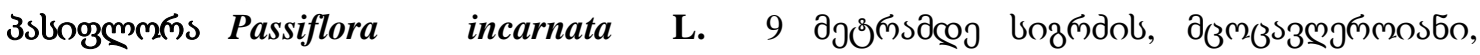

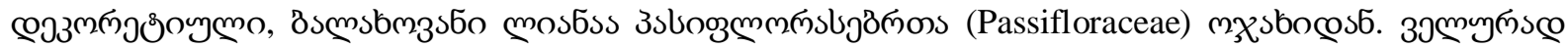

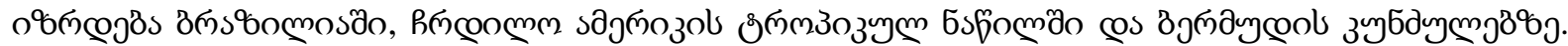

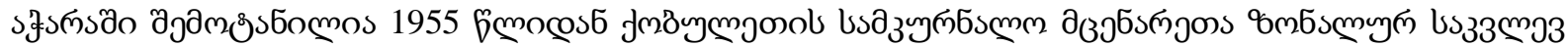

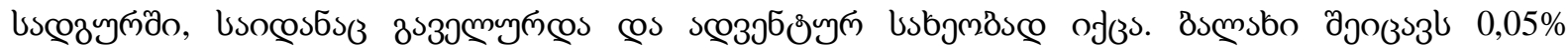

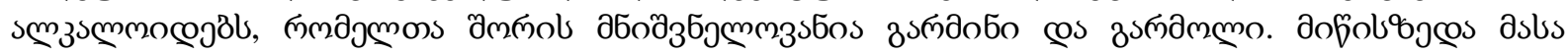

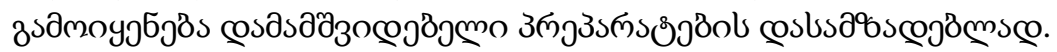

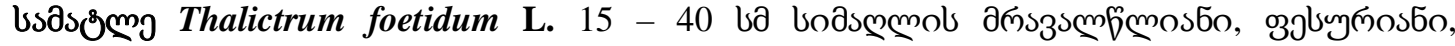

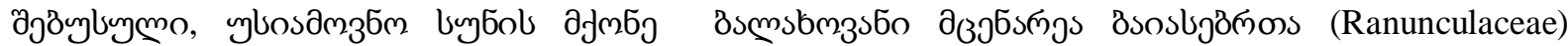

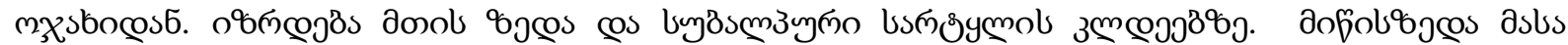

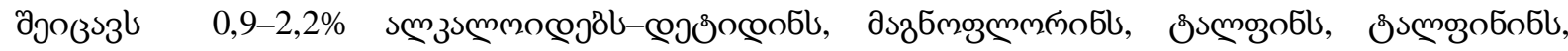

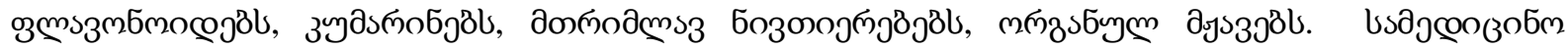

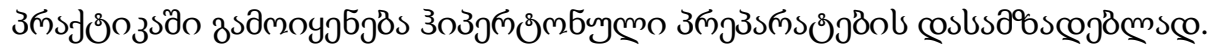

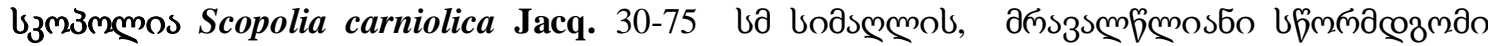

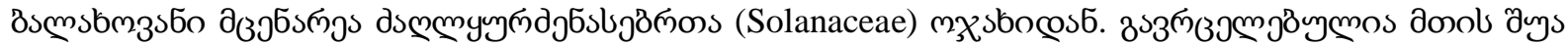

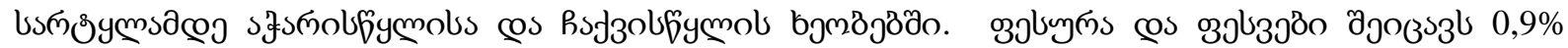

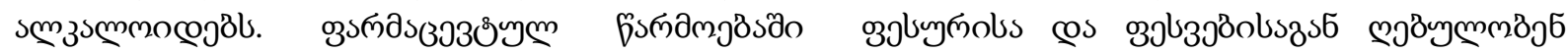

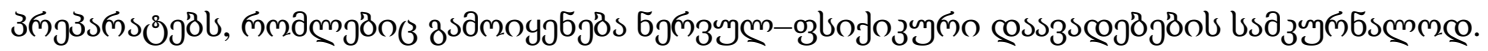

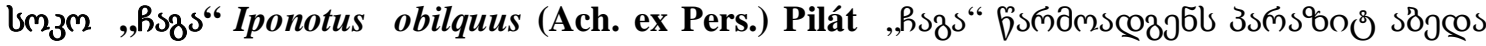

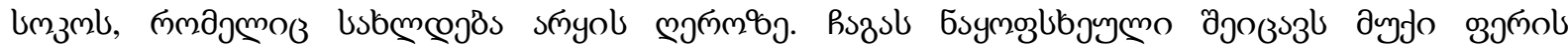

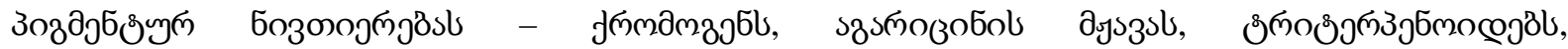

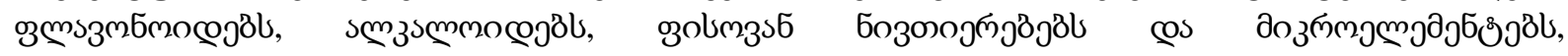

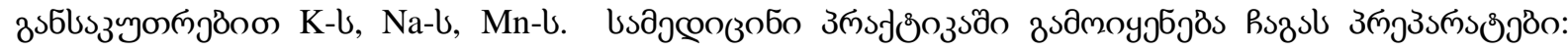

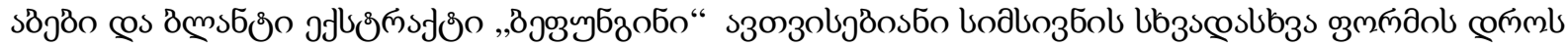

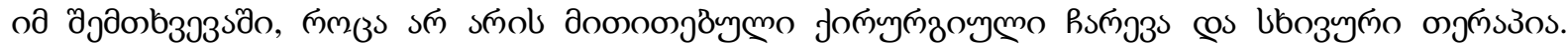

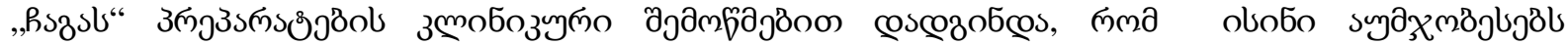

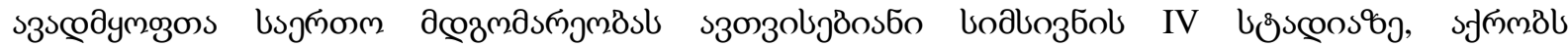

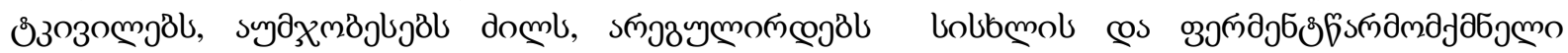

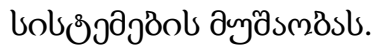

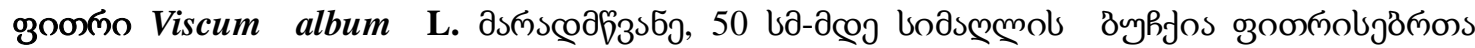

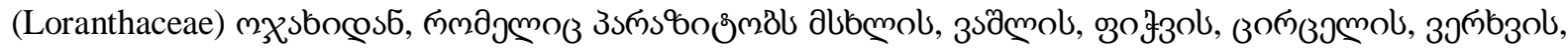

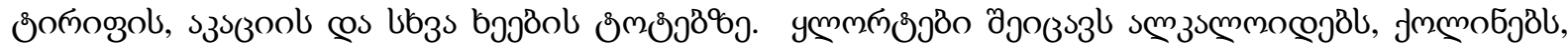

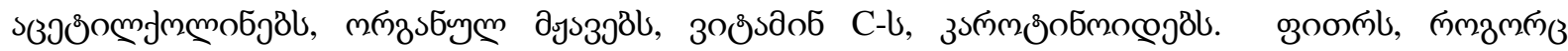

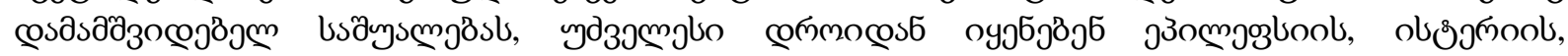

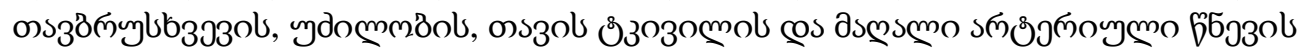

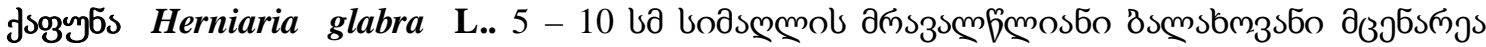

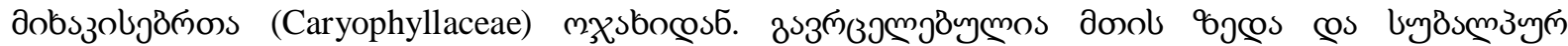

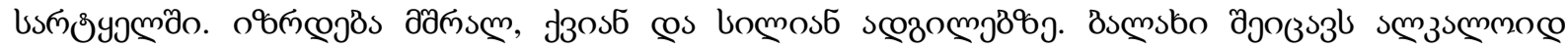

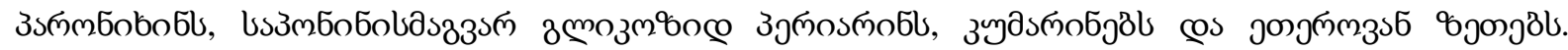

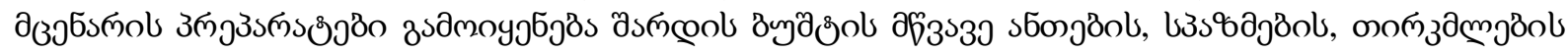

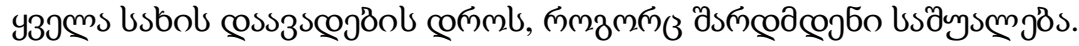

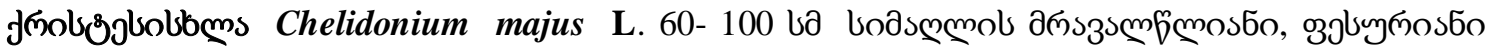

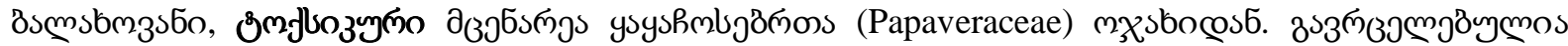

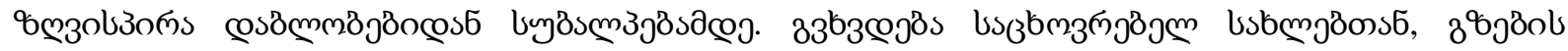

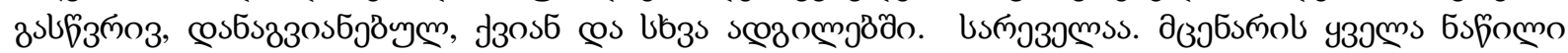

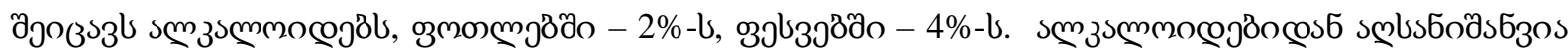




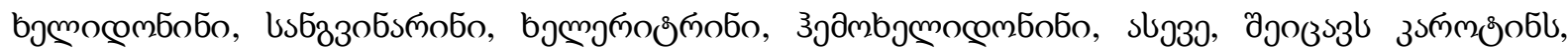

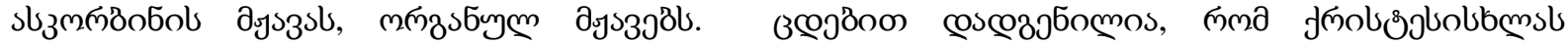

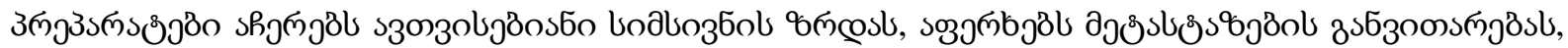

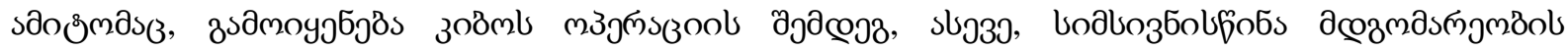

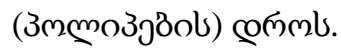

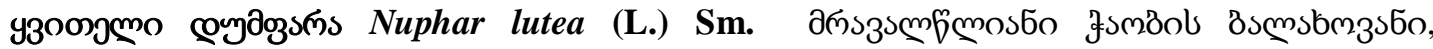

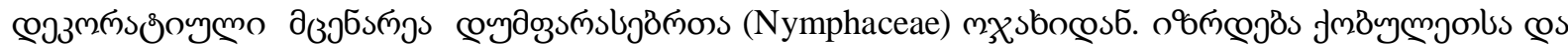

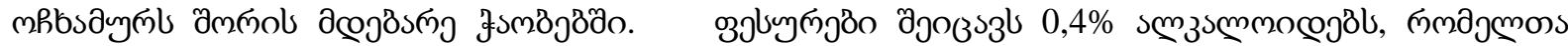

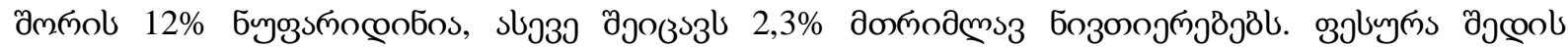

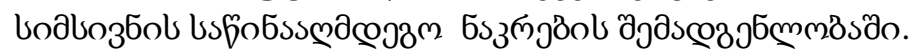

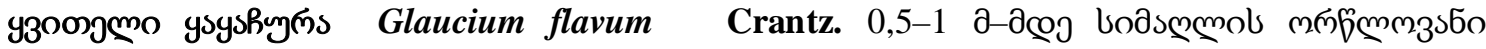

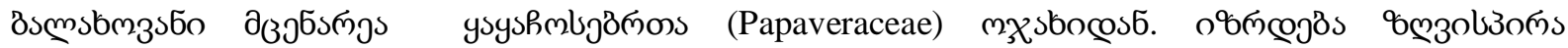

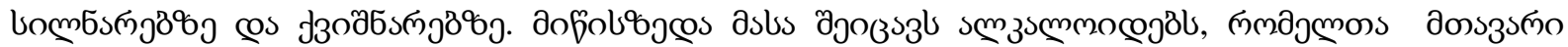

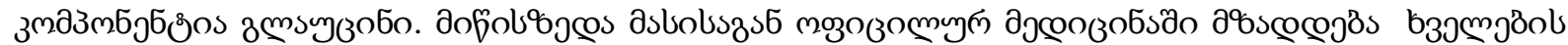

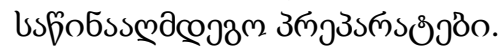

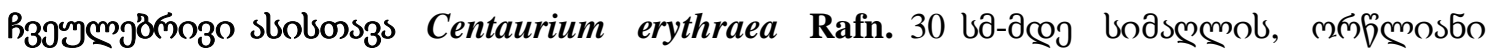

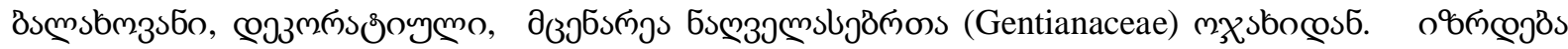

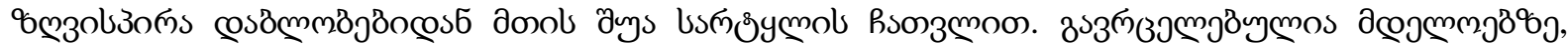

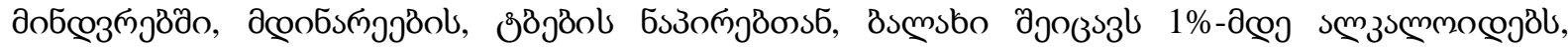

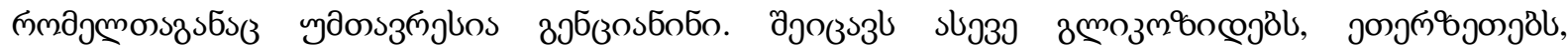

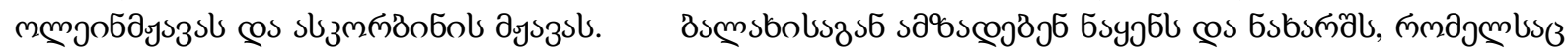
оујб

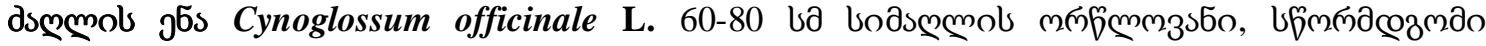

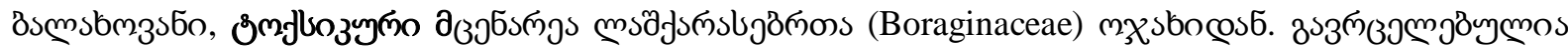

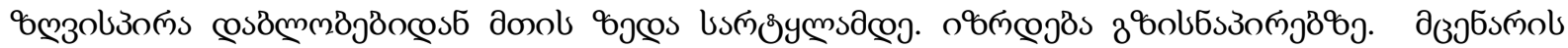

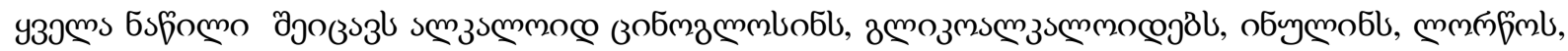

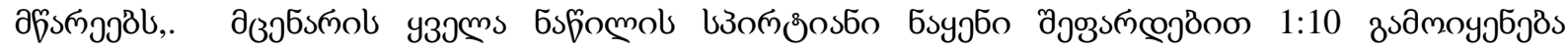

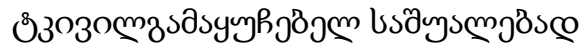

\section{зलоslo эком}

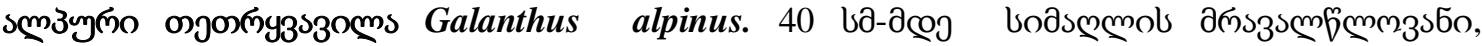

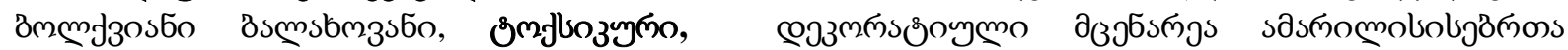

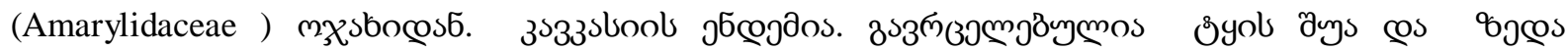

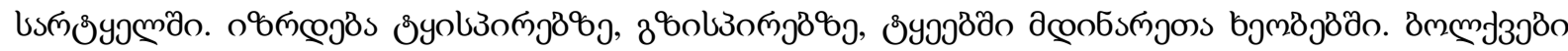

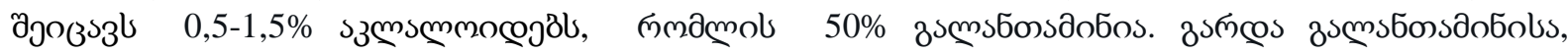

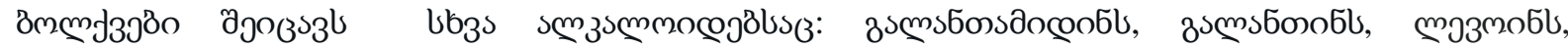

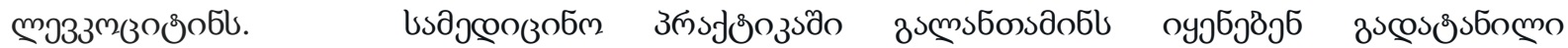

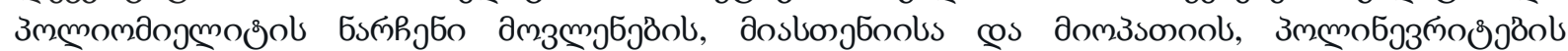

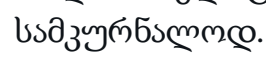

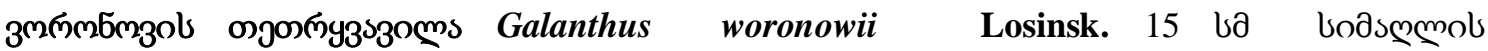

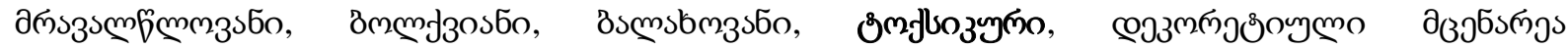

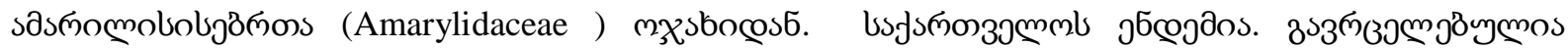

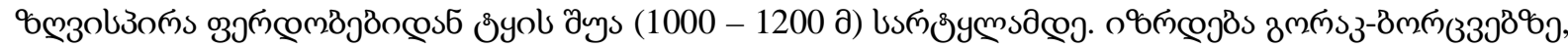

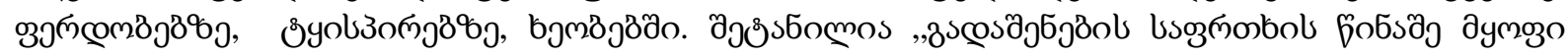

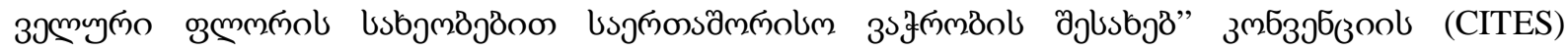

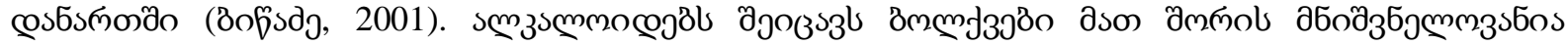

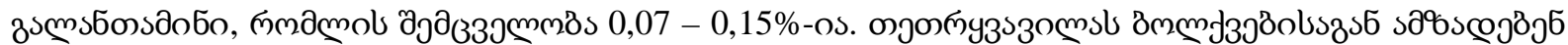

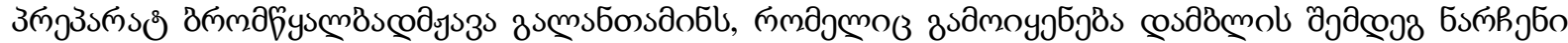

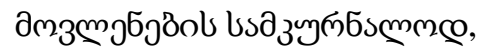

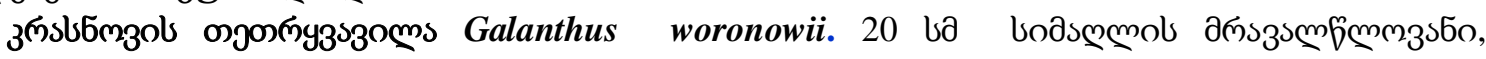

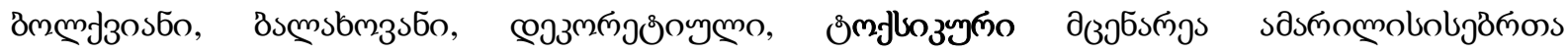




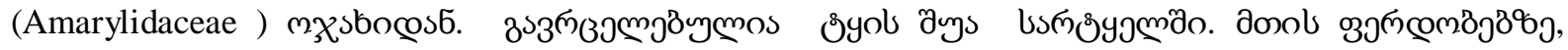

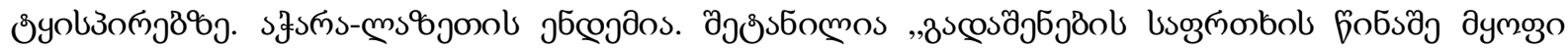

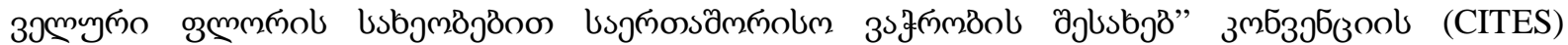

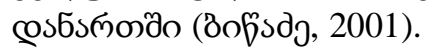

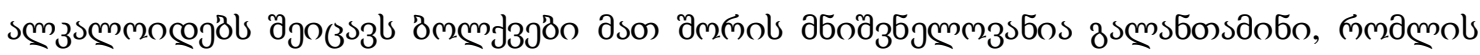

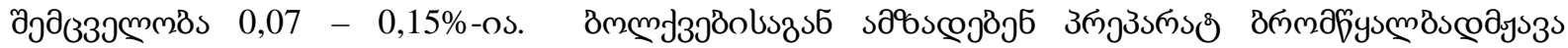

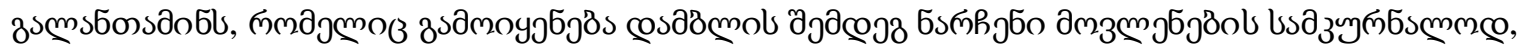

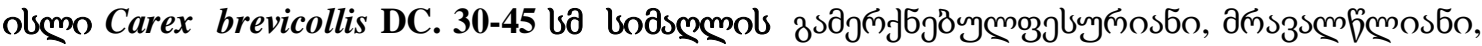

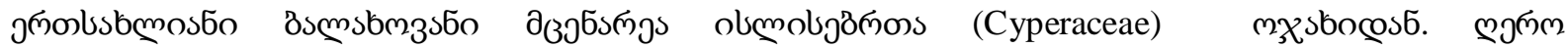

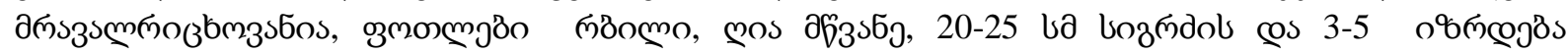

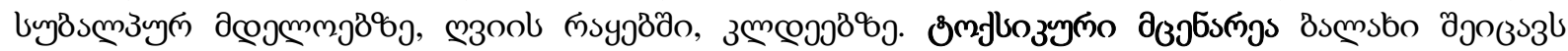

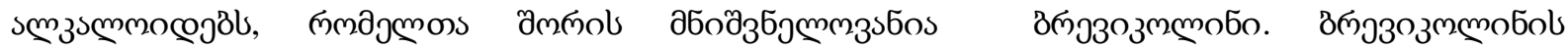

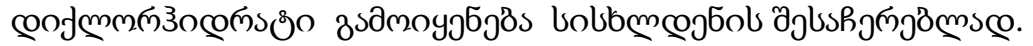

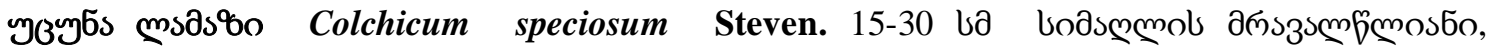

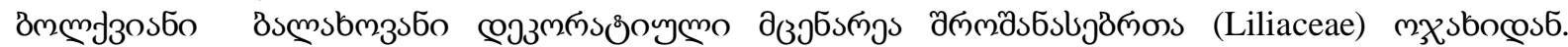

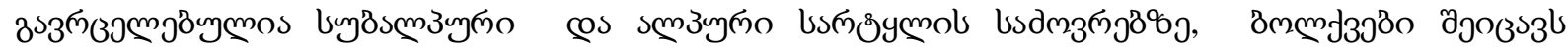

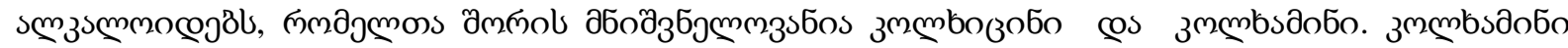

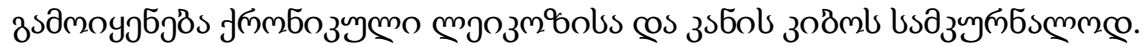

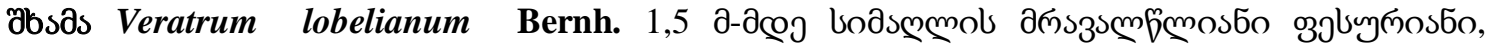

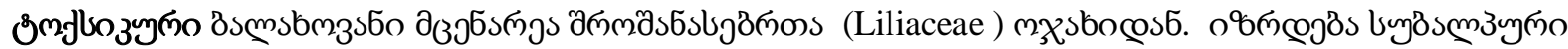

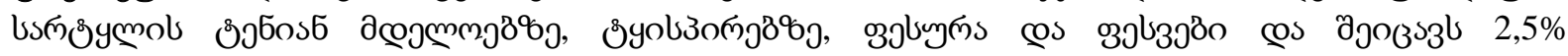

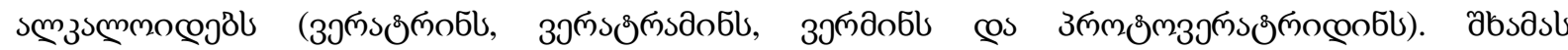

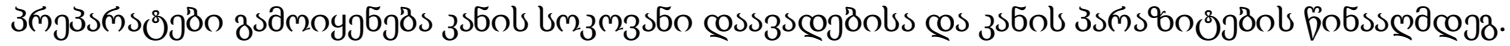

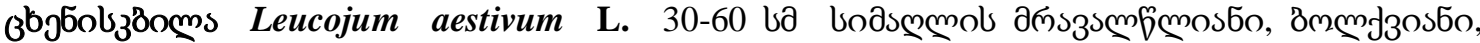

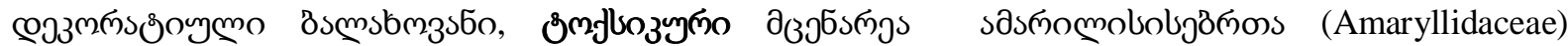

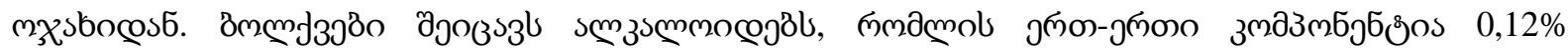

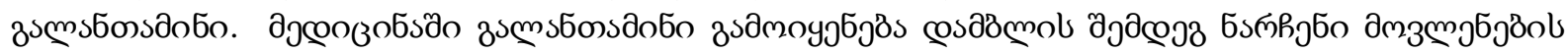

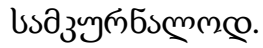

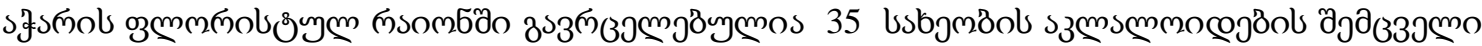

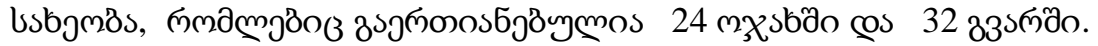

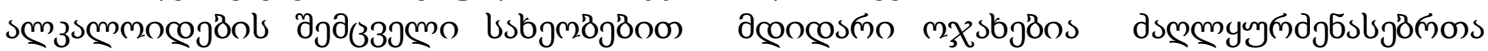
Solanaceae œos sasmomobobjzmons Amarillidaceae.

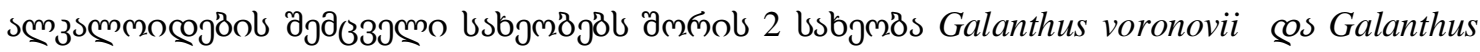

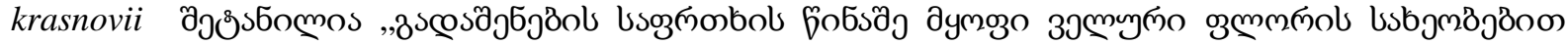

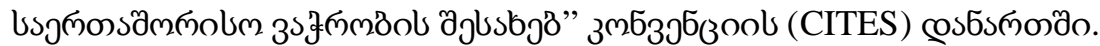

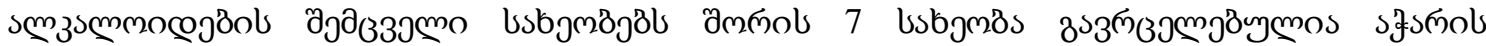

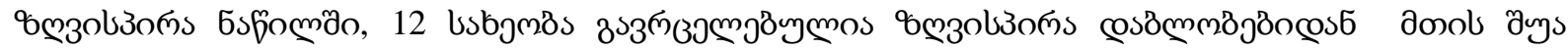
usলি

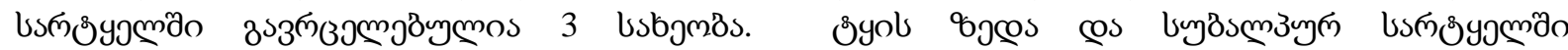

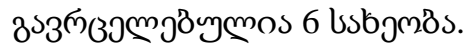

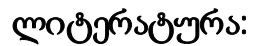

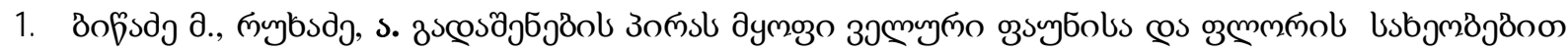

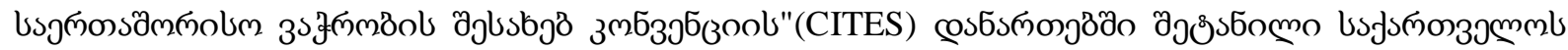

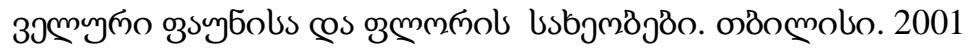

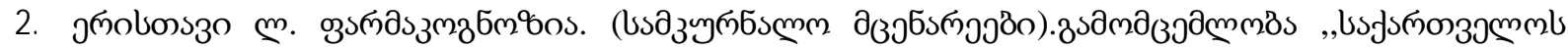

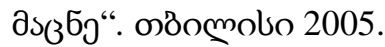




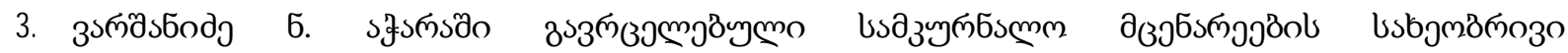

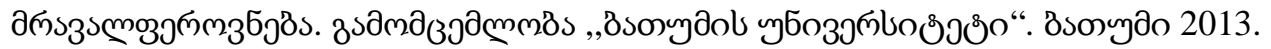

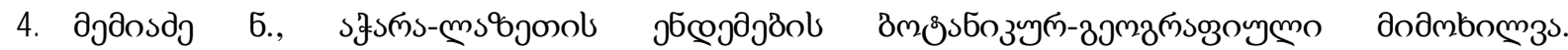

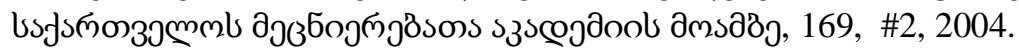

5. Дмитриева А.А. Определитель растений Аджарии. Тбилиси, „Мецниереба, т.1, 1990.

6. Дмитриева А.А. Определитель растений Аджарии. Тбилиси, „Мецниереба, т.II 1990.

7. Czerepanov S.K. 1995. Vascular plants of Russia and Adjacent states (the former USSR) //Cambridge University pressЧерепанов, 1995

8. Varshanidze, N., Turmanidze, N., Dolidze, K., Zarnadze, N., Diasamidze, I., Epitashvili, T., \& Katcharava, T. (2018). Biodiversity of Medicinal Plants Containing Essential Oil and Their Spreading in Adjara. Universal Journal of Agricultural Research 6(3): 99-104, 2018 http://www.hrpub.org DOI: 10.13189/ujar.2018.060301

9. Jakeli, E., Varshanidze, N., Diasamidze, I., Dolidze, K., \& Zarnadze, N. (2018). Biodiversity of Medicinal Plants of wild flora in Ajara-South Colchis and their usage in folk medicine. NWSA Academic Journals editors. 3rd International Science Semposium Proceeding Book. Turkey:Firat Akademi A. Ş, 80-96. 10. http://www.theplantlist.org/

\title{
Diversity of Plants Containing Alkaloids and Peculiarities of Distribution in Adjara
}

\author{
Varshanidze Natela, Diasamidze Inga, Turmanidze Nazi, \\ Dolidze Ketevan, Jakeli Eter, Bolkvadze Gia \\ SH ota Rustaveli State University,Batumi
}

\begin{abstract}
Alkaloids are nitrogen-containing organic compounds that are widely used in medicine for the treatment of cardiovascular, gastrointestinal, nervous, tumor pathologies. The aim of the research was to study the species diversity of alkaloid-containing plants, distribution areas, habitats and peculiarities of use in the floristic region of Adjara. According to the research, 35 species of alkaloids are distributed in the floristic region of Adjara, which are united in 24 families and 32 genera, including 28 species of the genus Orlebnia, 7 species of the genus Erlebnia, 1 species is representative of the fungal kingdom. Two families are rich in species containing alkaloids: dog Solanaceae and Amarillidaceae. According to life forms: 2 species of trees, 3 species of shrubs, 2 species of lianas, 27 species of perennial grasses. Among the studied species 4 species are endemic (Memiadze 2004), including one species is endemic to the Caucasus, 1 species is endemic to Georgia, one species is endemic to Adjara-Lazeti, and one species is endemic to Adjara. 2 species Galanthus voronovii and Galanthus krasnovii are included in the Annex to the Convention on International Trade in Endangered Species of Wild Fauna (CITES) (Bitsadze, 2001). Foreign flora Adventuria 2 species Gleditsia triacanthos Passiflora incarnata L 15 species are ornamental, 13 species are toxic.
\end{abstract}

Key words: M edicinal plant/herb, endemic, alkaloid

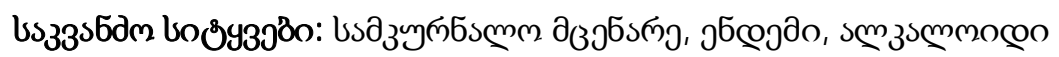

